

\title{
Representaciones en Resolución de Problemas: Un estudio de caso con problemas de optimización ${ }^{1}$
}

\section{José L. Villegas ${ }^{1}$, Enrique Castro ${ }^{2}$ y José Gutiérrez ${ }^{3}$}

\author{
${ }^{1}$ Universidad de Los Andes. Venezuela \\ ${ }^{2}$ Departamento de Didáctica de las Matemáticas. Universidad de Granada \\ ${ }^{3}$ Dpto de Métodos de Investigación y Diagnóstico en Educación. Universidad de \\ Granada
}

\section{España}

Dirección Postal: José Luis Villegas Castellanos. Dpto Didáctica de la Matemática. Facultad de Ciencias de la Educación. Universidad de Granada. España: joselovi@yahoo.es (C) Education \& Psychology I+D+i and Editorial EOS (Spain)

\footnotetext{
${ }^{1}$ Este trabajo ha sido realizado dentro del proyecto SEJ2006-09056 "Representaciones, nuevas tecnologías construcción de significados en Educación Matemática" financiado por el Plan Nacional de I + D + I del Ministerio de Educación y Ciencia y cofinanciado con fondos FEDER de la Comunidad Europea.
} 


\section{Resumen}

Introducción. Las representaciones juegan un papel fundamental en el pensamiento matemático, favorecen la comprensión de los conceptos matemáticos y estimulan el desarrollo de un pensamiento flexible y versátil en la resolución de problemas. En este trabajo nos ceñimos a problemas de optimización, de gran importancia y predicamento en la enseñanza y aprendizaje de la matemática a nivel superior

Método. Mediante metodología observacional presentamos los patrones de representación empleados por tres estudiantes de quinto curso de la licenciatura de matemáticas de la Universidad de Granada (España), obtenidos mediante el análisis de protocolos, en el que los registros escritos van acompañados de protocolos de pensar en voz alta. El instrumento empleado fue construido ad hoc y consta de tres problemas de optimización. Las sesiones de resolución de problemas fueron individuales, en un ambiente aislado y fueron grabadas en vídeo.

Resultados. Para el estudio de los datos se diseñó un marco para el análisis de protocolos con el que se investigan las transcripciones de las producciones de los sujetos. Los resultados se exponen en forma de análisis microscópico de caso en los que se pormenorizan los registros de representación empleados, las traducciones entre registros y el tiempo empleado en estas acciones, a partir de los cuales se realiza un perfil diferenciador de los resolutores. Según este perfil los tres participantes tienen una tipología distinta.

Discusión y Conclusiones. El marco para el análisis microscópico de los protocolos de resolución de problemas se ha mostrado adecuado para describir las representaciones y traducción entre representaciones en resolución de problemas. El proceso de segmentación y codificación nos ha llevado a considerar necesario incluir episodios calificados en principio como eventos no catalogables como representaciones. La caracterización de los resolutotes muestra la fuerte relación entre el éxito en la resolución de problemas de optimización y la habilidad en el manejo de las representaciones.

Palabras Clave: Resolución de problemas, representaciones, problemas de optimización, estudio de casos

Recibido: 12/11/08 Aceptación inicial: 01/12/08 Aceptación final: 26/01/09 


\section{Abstract}

Introduction. Representations play an essentials role in mathematical thinking. They favour the understanding of mathematical concepts and stimulate the development of flexible and versatile thinking in problem solving. Since the representations used are linked to the tasks, here we focus on optimization problems. These types of problems are considered important in mathematics teaching and learning in higher education.

Method. By using an observational methodology, we present the representation patterns employed by three students on fifth-year of the mathematics degree at the University of Granada (Spain). They were obtained through protocols analysis in which students' written production was complemented with their spoken thinking protocols. The instrument used was intentionally built for this study and was composed by three optimization problems. Students solved the instrument individually in an isolated environment while being video-recorded.

Results. We designed a framework for the analysis of the protocols and used it to analyze the transcriptions of students' productions. As a microscopic analysis of particular cases, we describe the representation records used by the students, the translation of the records and the time employed in those actions. These elements are used to distinguish each solver's profile which happened to be different.

Discussion and conclusions. The designed framework for the microscopic analysis of the problem solving protocols was useful to describe the representations and translation between them in the solving problem process. The segmentation and codification process led us to consider necessary to include episodes which initially were not identified as representations. The solvers' characterization displays a strong connection between students' success on optimization problem solving and their skills at using representations.

Keywords: problem solving, representations, optimization problems, case studies.

Received: 11/12/08 Initial Acceptance: 12/01/08 Final Acceptance: 01/26/09 


\section{Introducción}

Una característica de la inteligencia humana es el uso de diferentes tipos de representación, con fines lúdicos, normativos, comunicativos, simbólicos, artísticos, literarios, musicales. Esta característica nos diferencia de los animales y de la inteligencia artificial y es quizá una de las razones que justifica el hecho de que la investigación sobre el lugar de las representaciones en el aprendizaje de las matemáticas y en la resolución de problemas haya experimentado un crecimiento importante en los últimos años. Como resultado de estas investigaciones se considera indiscutible la importancia de las múltiples representaciones en el desarrollo del pensamiento matemático (Brenner et al. 1997; Cuoco y Curzio, 2001), de cuya evidencia principal dan cuenta las agendas de prioridades establecidas en comités y reuniones científicas de rango internacional (Goldin, 1998b; Hitt, 2002).

El término representación es complejo y está abierto a muchas interpretaciones (Rico, 2000). En este estudio el término representación se refiere a todas aquellas formas con que hacemos presentes los objetos o procesos matemáticos, y nos es esencial para definir, explicar, visualizar, registrar y comunicar el conocimiento matemático.

Los sistemas de representación reúnen unos requisitos de complejidad, interrelación y poder de simbolización y abstracción cuyo dominio amplía y enriquece la inteligencia humana en cuanto son instrumentos útiles de modelización de la realidad y herramientas prácticas para la resolución de diferentes tipos de problemas de la vida real. Mediante diversos medios de expresión los seres humanos nos familiarizamos y aprendemos un sin fin de códigos, símbolos, señales, iconos y lenguajes de diversa naturaleza. El poder heurístico y de comunicación que nos dan esos elementos representativos a la actividad humana aumenta en la medida en que dichos medios de expresión se integran formalmente en sistemas complejos de simbolización sometidos a reglas sintácticas y gramaticales.

La importancia de las representaciones se pone de manifiesto en los trabajos de Duval (1998), para quien no es posible estudiar los fenómenos relativos al conocimiento sin recurrir a la noción de representación, porque no hay conocimiento que un sujeto pueda movilizar sin una actividad de representación, de tal forma que las representaciones en la matemática, y su enseñanza, son fundamentales, pues sus objetos de estudio son construcciones de la mente y requerimos de representaciones para interactuar con ellos. 
En este trabajo se describen las representaciones usadas por un grupo de universitarios cuando resuelven un tipo específico de problemas de optimización cuyas posibilidades de representación y modelización de situaciones es múltiple y variada. Se han empleado protocolos de pensamiento en voz alta y para su análisis se ha construido un marco empírico inspirado en los trabajos de Schoenfeld (1985). Con esta herramienta se analizan en este trabajo las estrategias de representación, el tiempo invertido en cada representación y los mecanismos de traducción de un sistema a otro.

\section{Importancia de las Representaciones en la Resolución de Problemas}

A lo largo de las dos últimas décadas se ha puesto de manifiesto el elevado consenso que existe en la comunidad investigadora sobre el uso de las representaciones por parte de los estudiantes y su éxito como instrumento al servicio de la resolución de problemas (Castro, 2008; DeBellis y Goldin, 2006). Además, las múltiples representaciones pueden ser utilizadas para desarrollar de manera más profunda y flexible la comprensión de conceptos y procesos; (Cuoco, y Curcio, 2001; Hiebert y Carpenter, 1992; Kaput, 1987; Koedinger, y Nathan, 2004). Si bien, es importante poseer varias representaciones de un concepto, la pura existencia de estas no es suficiente para permitir un uso flexible del concepto en resolución de problemas. Para manejar con éxito la información utilizada en la resolución de problemas, las representaciones deben ser correctas y estar fuertemente vinculadas: "uno necesita tener la posibilidad de cambiar de una representación a otra, cuando la otra sea más eficiente para el nuevo paso que queremos dar" (Dreyfus, 1991).

Dada la importancia de la resolución de problemas en educación (NCTM, 1989, 2000, 2006) la investigación centrada en el estudio de las representaciones que se forman los estudiantes cuando resuelven problemas es un tópico de interés tanto desde el punto de vista educativo como investigacional. El National Council of Teachers of Mathematics (NCTM) reconoce que el buen uso de múltiples representaciones aporta un conjunto flexible de herramientas para resolver problemas y para apreciar la consistencia y la belleza de la matemática. Entre los objetivos de la enseñanza en el nivel medio superior destaca el que los alumnos logren tener un buen conocimiento de las distintas formas de representar, puedan articular sin contradicciones estas representaciones y recurran a ellas en forma espontánea durante la resolución de problemas ya que esto es clave y esencial para su éxito (Hitt, 1996). 
En este sentido, Cifarelli (1998) pone de manifiesto que el éxito de los resolutores de problemas competentes puede ser debido en gran parte a su habilidad para construir representaciones apropiadas para situaciones de resolución de problemas, y es que la elección, por parte de los estudiantes, de representaciones apropiadas les brindará la oportunidad de aprender a sopesar las ventajas y desventajas de las diferentes formas de representación (Schultz y Waters, 2000), y a emplearlas como herramientas para la resolución de problemas. Por su parte, Lesh, Post y Behr (1987) describen el papel que juegan las representaciones, y la traducción entre representaciones, en el aprendizaje de las matemáticas y la resolución de problemas. El término representación lo emplean en sentido restringido, como expresiones externas de conceptualizaciones internas de los estudiantes. Para estos investigadores, los distintos sistemas de representación no sólo son importantes por "méritos propios", sino que las traducciones entre ellos y las transformaciones dentro de ellos son también importantes, así, la habilidad en la traducción (o la falta de esta) es un factor significativo que afecta tanto al aprendizaje como al rendimiento en la resolución de problemas.

La importancia de la actividad de conversión o traducción de un sistema de representación a otro, partiendo de enunciados verbales en formato de narración escrita es destacada por Duval (1993, 1998, 2006), quien, subraya la necesidad de desarrollar investigaciones en esa dirección. Todas las tareas seleccionadas para el estudio que aquí se describen se ajustan a las recomendaciones anteriores, por cuanto que: son enunciados verbales cuya resolución se inscribe en el campo matemático, admiten el uso de más de un sistema de representación como vías factibles de estructuración, visualización y búsqueda de solución; asimismo, responden a una tipología especifica de enunciados problema de matemáticas denominados de optimización.

\section{Problemas de optimización. Razones para su empleo en el estudio}

En los estudios previos sobre representaciones y resolución de problemas se ha puesto de manifiesto que hay una fuerte asociación entre los tipos de representaciones que ponen en juego los resolutores y el tipo de tareas que se les platean (Castro, et al., 1999). Por ello, es conveniente realizar estos estudios con grupos de problemas que respondan a una unidad conceptual de contenido matemático. En nuestro trabajo nos hemos centrado en los problemas de optimización, que conforman un bloque importante en la educación matemática. 
El deseo de optimizar es inherente a los humanos. La búsqueda de extremos inspira a alpinistas, científicos, matemáticos, y al ser humano en general. Los métodos de optimización exploran suposiciones sobre el carácter de respuesta de la función objetivo, variando parámetros y sugiriendo el mejor camino para cambiarlos. La variedad de suposiciones a priori corresponde a la variedad de métodos de optimización.

Para explicar cuan difíciles son los problemas de optimización, podemos observar su clasificación en la figura 1. Muchos métodos de optimización se diseñan para diverso número de controles independientes (dimensionalidad). Estos rangos corresponden a una o más variables.

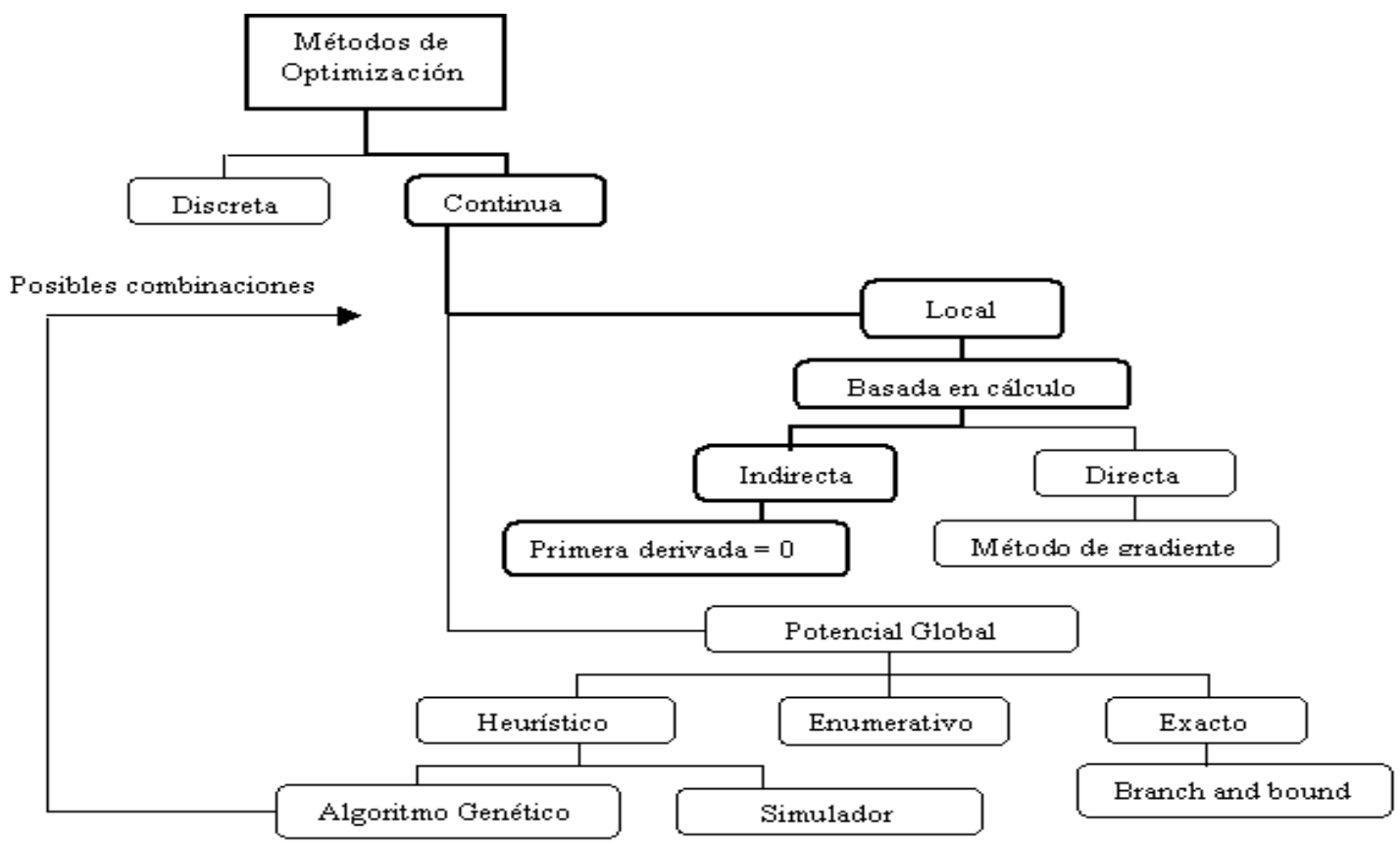

Figura 1. Árbol de optimización

Los problemas de optimización que usaremos en este estudio, son los que siguen la ruta en negrita de la figura 1, y en los cuales intervenga una sola variable independiente. Este tipo de problemas se encuentran entre las aplicaciones más frecuentes y llamativas del cálculo, además, la vida cotidiana está llena de aplicaciones prácticas en las que intervienen este tipo de problemas. Por todas estas razones, hemos elegido este tipo de problemas en nuestro estudio: por constituir un campo de problemas matemáticos bien delimitado desde el punto de vista conceptual, por su alto grado de aplicabilidad y por su potencial intrínseco en el uso de varias formas de representación para su resolución. 
Los problemas de optimización que se han seleccionado son, en concreto problemas de enunciado verbal y relacionados con el mundo real. De tal manera que la representación de partida es un enunciado narrativo o texto sobre el que es necesario realizar una traducción para construir una estructura matemática; este proceso de representación tiene una importancia particular en la matemática aplicada y un gran peso en la universidad y más recientemente en el currículo escolar y se le llama modelización. El término modelización se refiere a la búsqueda de una representación matemática para un objeto o proceso no matemático; en este caso, se trata de construir una estructura matemática o teoría, la cual incorpora características esenciales del objeto, sistema o proceso a describir. Esta estructura-modelo, puede ser, a su vez, usada para estudiar la conducta del objeto o proceso que es modelado (Dreyfus, 1991).

El proceso de representar es, hasta cierto punto, análogo al proceso de modelizar, pero se sitúa en otro nivel, ya que en una modelización la situación o sistema con el que se trabaja es físico, y el modelo es matemático; mientras que en una representación el objeto a ser representado es la estructura matemática, y el modelo es una estructura mental. Por consiguiente, la representación mental está relacionada con el modelo matemático como el modelo matemático está relacionado con el sistema físico (Dreyfus, 1991). Cada uno es una traducción parcial del otro, cada uno refleja algunas propiedades, aunque no todas, del otro; y cada uno mejora la capacidad para manipular mentalmente el sistema bajo consideración.

Los problemas de optimización han sido usados en múltiples investigaciones relacionadas con las representaciones y la resolución de problemas (Camacho y González, 1998; Campos y Estrada, 1999; Porzio, 1999; Shoenfeld, 1985). Asimismo este tipo de problemas son de gran importancia en el currículo de matemática de los últimos años de secundaria y primeros de universidad de carreras de ciencias, ingeniería, economía y finanzas.

El valor e interés formativo de los problemas de optimización ha sido reconocido ampliamente en los Diseños Curriculares de Matemáticas para los estudiantes entre 16 y 18 años de la Junta de Andalucía (1989), admitiendo que los problemas de optimización son de gran interés en el proceso de enseñanza y aprendizaje de matemáticas porque:

- Muestran aplicabilidad y utilidad para el análisis y la resolución de cuestiones prácticas de carácter muy diverso.

- Ponen de manifiesto la potencia del cálculo diferencial, que proporciona un 
método general para abordar los problemas extremos.

- Suscitan situaciones muy apropiadas desde el punto de vista formativo para ejercitarse en la heurística como procedimiento valioso para abordar la resolución de problemas.

- Generan una motivación añadida basada en su poder aplicado y en la creatividad que requiere el uso múltiple de representaciones.

Un uso generalizado de este tipo de problemas se ha venido realizando en situaciones de aplicación de los conceptos del cálculo, y reducido a unos niveles avanzados de dominio conceptual de aspectos matemáticos. Con el uso de sistemas de representación aplicados a estos ámbitos de la educación matemática se amplían las posibilidades de trabajo a niveles inferiores que no requieran un dominio experto del contenido.

Objetivos del estudio

En este estudio nos proponemos como objetivo general describir las representaciones y los procesos de traducción entre representaciones en la resolución de problemas de optimización, que emplean un grupo de estudiantes universitarios.

Entre los objetivos específicos en que se desglosa el estudio podemos destacar:

1. Determinar qué tipo de representaciones utilizan los resolutores expertos en la resolución de problemas de optimización.

2. Evaluar cómo influye el tiempo empleado en cada representación y en la traducción entre representaciones en el éxito de los resolutores en la resolución de problemas de optimización.

3. Examinar si existen regularidades en el empleo de las representaciones ó en la traducción entre representaciones al resolver problemas de optimización.

4. Caracterizar a los resolutores de acuerdo a las representaciones y traducción entre representaciones usadas en las diferentes situaciones problema.

Para tal fin, los tipos de representaciones externas que se tomaron en cuenta fueron las siguientes:

1. Representación verbal del enunciado del problema: consiste fundamentalmente en el 
enunciado del problema, que puede ser escrito o hablado.

2. Representación pictórica: aquella que se hace a través de dibujos, diagramas ó gráficos, así como cualquier tipo de acción relacionada con estos.

3. Representación simbólica: se refiere a la que se forma de números, signos de operación y de relación; símbolos algebraicos, además de cualquier tipo de acción referida a estos.

Además de las traducciones entre ellas (fig. 2).

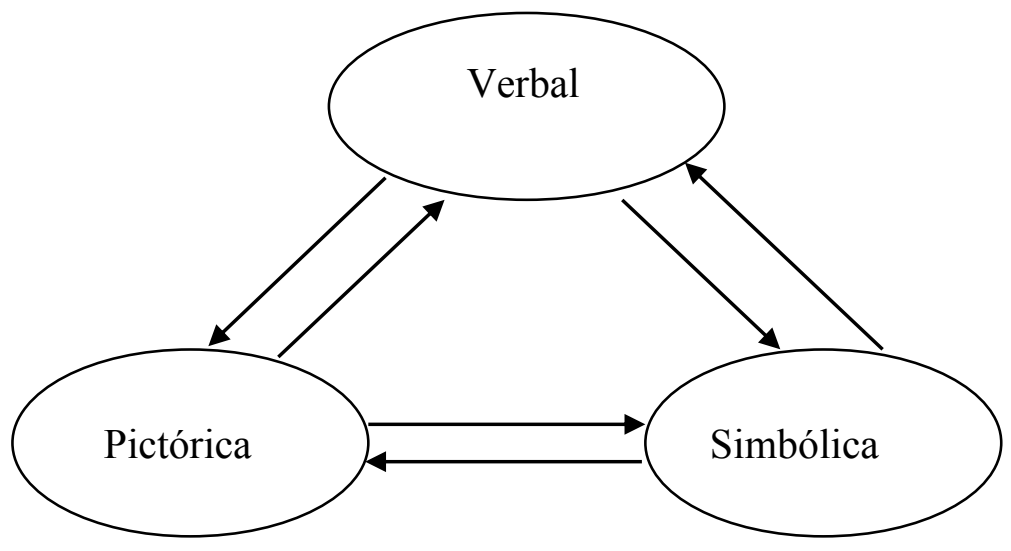

Figura 2. Tipos de Sistemas de Representación

Una dificultad inicial en el estudio, para cumplir con el objetivo general, radicó en el hecho de la inexistencia de un marco para el análisis de protocolos en resolución de problemas de optimización que tomara en cuenta las representaciones y la traducción entre representaciones; por tal razón fue necesaria su elaboración, la cual la hicimos adaptando el análisis de protocolos realizado por Schoenfeld (1985).

En particular, las cuestiones de interés se centran en describir las diferencias y similitudes en cuanto a la variable tiempo en cada representación y en cada traducción entre representaciones, empleada en función del tipo y perfil del resolutor; así como valorar la potencialidad representacional de los diferentes tipos de problemas de optimización seleccionados así como la versatilidad en su resolución por distintos estudiantes expertos.

El estudio se sitúa en la tradición de los trabajos que consideran algunas de las dimensiones de las representaciones como elementos determinantes en la resolución de problemas (Ballard, 2000; Cifarelli, 1998; Janvier, 1987; Lesh, Post y Behr, 1987; Porzio, 1999). 


\section{Método}

\section{Participantes}

El trabajo puede considerarse un estudio de caso intrínseco en los términos que lo define Stake (1999) y Gutiérrez et al. (2002): estudio de la particularidad y la complejidad de uno o varios casos singulares, para llegar a comprender su actividad en unas determinadas circunstancias; y en la acepción dada por Marcelo (1991), "en cuanto examen intensivo o completo de una faceta o dimensión a lo largo del tiempo". Los sujetos que han sido objeto de este estudio de casos han sido tres estudiantes de quinto año de la Licenciatura de Matemáticas, especialidad Metodología de la Facultad de Ciencias de la Universidad de Granada del curso 2001-2002.

\section{Instrumentos}

El estudio ha seguido las directrices de la metodología observacional (Anguera et al, 1995), en orden a las recomendaciones dadas por Dane (1990) acerca del método observacional en cuanto procedimiento sistemático de investigación mediante el cual los eventos son seleccionados, registrados y codificados en unidades significativas de análisis. En concreto, la técnica empleada para la recogida de datos ha sido el análisis de protocolos, entendiendo por protocolo la descripción ordenada cronológicamente de las conductas que un sujeto desarrolla mientras ejecuta una tarea de resolución de problemas, siendo datos objeto de análisis tanto las verbalizaciones que realizan durante la resolución, como sus anotaciones, representaciones, secuencias y estrategias desplegadas en el proceso resolutor; acompañadas de algunos comentarios y observaciones del investigador (Schoenfeld, 1985). Interesa, por tanto analizar no solo las representaciones semióticas que explícitamente expresa el estudiante, sino también las representaciones mentales inferidas a través de éstas y de la conducta, al invitar al sujeto a que emplee la técnica de pensamiento en voz alta (thinking aloud), ya que hay un fuerte incremento en la cantidad de conducta que puede ser observada cuando la comparamos con el mismo sujeto en condiciones de silencio. Además, no es necesario que el sujeto sea previamente entrenado antes para ser capaz de pensar en voz alta en este tipo de tareas de resolución de problemas, y se puede inferir que este reporte verbal es consistente con la estructura de sus procesos cognitivos normales (Ericsson y Simon, 1993). 
Construcción y organización de la sesión de resolución de problemas

El primer paso, ante la inexistencia de un instrumento contrastado para la recogida de datos, fue realizar un estudio previo para la elaboración de un cuestionario definitivo, a la par queríamos examinar otros parámetros importantes, como el tiempo necesario para la solución de los problemas, el orden y la forma en que iban a ser planteados; el tipo de herramientas tecnológicas, que se adecuaban mejor a nuestros propósitos, para la recogida de datos de la sesión de resolución de problemas, así como encontrar cualesquiera otros aspectos que resultaran de interés para ser tomados en cuenta en el estudio definitivo.

En principio se construyó una batería de problemas de optimización siguiendo los siguientes criterios: que estuvieran en los libros de textos de nivel universitario, que fueran adecuados para estudiantes de primer año de la Universidad de carreras como Ciencias e Ingeniería, que el enunciado fuera estrictamente "narrativo", que fueran problemas matemáticos del mundo real, que no fuera necesario el uso de calculadoras, que necesitaran para su resolución el empleo de varias representaciones.

Se obtuvieron 47 problemas al seguir los requisitos arriba señalados; éstos se sometieron a consideración de algunos investigadores y licenciados en matemáticas que opinaron acerca de la pertinencia de estos en el estudio a realizar y si eran adecuados para los sujetos a participar en el estudio. Con estas opiniones se seleccionaron los cuatro problemas, con los que se efectuó una prueba piloto, que fue aplicada a tres voluntarios, licenciados en matemática que tenían entre dos y seis años de graduados. Resolvieron los problemas en voz alta y la sesiones de resolución de problemas fueron grabadas en audio.

Con los resultados obtenidos de la aplicación de la prueba piloto el número de problemas se vio reducido a tres, ya que uno de los problemas fue considerado como "complicado" y no congruente con los otros. No fue necesario sustituir este problema ya que se estimó que cuatro problemas serian demasiados para ser resueltos en una sola sesión. Asimismo, el tiempo necesario para cada problema se estableció en 20 minutos, de tal forma que el tiempo total para cada resolutor sería, de aproximadamente, 90 minutos, 60 para la resolución de los problemas y 30 minutos para que el resolutor diera sus comentarios finales acerca de cada problema y al proceso en general. También encontramos que era estrictamente necesario que las 
sesiones fueran grabadas en vídeo y apoyadas por grabaciones magnetofónicas, las cuales reforzarían el sonido de la vídeo grabadora

Tomando en cuenta la información aportada por la prueba piloto, los problemas elegidos para nuestro estudio definitivo son los siguientes:

Tabla 1. Problemas propuestos

PROBLEMA 1: A un lado de un río recto de $1 \mathrm{Km}$. de anchura hay una central eléctrica y al otro lado $5 \mathrm{Km}$. corriente arriba una factoría; su dueño desea tender un cable desde la central eléctrica hasta la factoría, él sabe que tender cable por tierra cuesta 3 euros por metro y tenderlo por agua cuesta 5 euros por metro. ¿Cuál será la trayectoria del tendido que le resulta más económica. Si el cable por tierra tiene el mismo precio que el cable por agua. ¿Cuál será la trayectoria?

PROBLEMA 2: Se desea construir una ventana rectangular coronada por un semicírculo (el ancho del rectángulo ha de ser igual que el del semicírculo). Cuál es la ventana que admitirá la mayor cantidad de luz posible, si su perimetro ha de ser fijo.

PROBLEMA 3: Un espejo rectangular de dimensiones 80 y $90 \mathrm{~cm}$. se rompe por una esquina en línea recta. De los dos trozos que quedan, el menor tiene una forma de triángulo rectángulo de catetos 10 y $12 \mathrm{~cm}$., correspondientes, respectivamente, a las dimensiones menor y mayor del espejo. ¿Cuál es el espejo rectangular más grande que se puede obtener del trozo mayor?

Los problemas seleccionados tenían las siguientes características:

Tabla 2. Características de los problemas

\begin{tabular}{ccccc}
\cline { 2 - 5 } & $\begin{array}{c}\text { Tipo de pro- } \\
\text { blema }\end{array}$ & $\begin{array}{c}\text { Tipo de opti- } \\
\text { mización }\end{array}$ & $\begin{array}{c}\text { Funciones } \\
\text { involucradas }\end{array}$ & $\begin{array}{c}\text { Números in- } \\
\text { volucrados }\end{array}$ \\
\hline Problema 1 & $\begin{array}{c}\text { En dos me- } \\
\text { dios }\end{array}$ & Costo mínimo & $\begin{array}{c}\text { Lineal } \\
\text { Radical }\end{array}$ & $\begin{array}{c}\text { Enteros gran- } \\
\text { des } \\
\text { Fraccionarios }\end{array}$ \\
Problema 2 & $\begin{array}{c}\text { Minimizar } \\
\text { construcción }\end{array}$ & Área mínima & Lineal & Fraccionarios \\
Cuadrática & Irracionales \\
Problema 3 & $\begin{array}{c}\text { Inscribir/ } \\
\text { circunscribir }\end{array}$ & Área máxima & Lineal & Enteros gran- \\
& & & des \\
& & & Fuadrática & \\
\hline
\end{tabular}

Procedimiento

Los estudiantes tenían que resolver los tres problemas de optimización presentados en el apartado anterior. Estos problemas eran facilitados a los sujetos individualmente, cada uno 
en un folio y escritos con letra "grande", con el fin de tener una mejor observación de los problemas a la hora de transcribir los datos y no se les daba el siguiente hasta no haber terminado y entregado el anterior.

Las sesiones grabaciones en vídeo comenzaron a las 9:30 y el número de personas que se encontraban en la sala era muy reducida. Además del resolutor, se encontraban el investigador y un asistente de investigación, encargado de ayudar con los equipos de vídeo y de audio, el hecho de limitar el número de personas en la prueba se hizo con la finalidad de que existiera una influencia mínima en el comportamiento del resolutor. El investigador y el asistente actuaron como observadores, en el proceso anotando en un cuaderno lo que consideraban importante de ser reseñado y las cuestiones que pensaban no quedarían reflejadas en la vídeo grabación.

Los problemas planteados fueron resueltos en voz alta, tratando que el investigador interviniera lo menos posible en el proceso de resolución, ya que para obtener y describir las actividades que constituyen la resolución de problemas, los datos se deben obtener desde una situación en la cual el sujeto trate con problemas estimulantes (en nuestro caso, problemas de optimización) y en el cual haya una mínima intervención del investigador (Gindburg et al., 1983). Las pocas intervenciones efectuadas ocurrían cuando el resolutor se quedaba en silencio durante un período largo de tiempo, o estaba "dándole vueltas" al problema sin encontrar salida y el investigador consideraba, que era más importante para su estudio, "encaminarlo" hacia la solución, ya que esto produciría mayor información.

Antes de iniciar la resolución de los problemas propuestos, el investigador informó las siguientes cuestiones relacionadas con la investigación y el proceso de resolución de problemas: la importancia futura del estudio para el proceso de enseñanza y aprendizaje, el por qué fue invitado a participar en el estudio, que sería grabado en vídeo mientras resuelve la prueba, el tiempo aproximado que le llevarían las tareas, la importancia de tomarse en serio la actividad, la necesidad de expresar en voz alta sus pensamientos desde que iniciara hasta que finaliza el problema planteado, la necesidad de verbalizar todos los pasos mientras estuviese resolviendo el problema, sin presuponer que algún pensamiento o paso es obvio o insignificante de verbalizar, que no borrara nada de lo escrito y si esto era necesario, que lo tachase preferiblemente, que los resultados de las tareas no repercutirían no influirán en los resultados académi- 
cos de las asignaturas cursadas en la Universidad, y que la información suministrada sería estrictamente confidencial.

El hecho de informar al resolutor lo antes expuesto tenía como finalidad, además de que conociera como actuar mientras resolvía los problemas, que se estableciera una buena relación investigador-resolutor de tal forma que estuviera tranquilo, para que su estado de animo, influyera lo menos posible en el proceso de resolución de los problemas.

\section{Análisis de Datos}

Para realizar las transcripciones el investigador observó atentamente el vídeo una primera vez, sin tomar ninguna nota por escrito, con el fin de familiarizarse con la voz y los gestos de cada resolutor, posteriormente realizó una primera transcripción con la cual se elaboró un texto escrito. Un segundo investigador efectuó paralelamente la transcripción y también elaboró un texto escrito. Ambos textos escritos fueron confrontados y puestos un común observando de nuevo el vídeo y corrigiendo lo que fuera necesario para obtener una trascripción definitiva.

Posterior a esta transcripción se procedió a la segmentación del texto en diferentes ítem, para esto se tomo como primer criterio que cada ítem corresponde a verbalizaciones que se hayan producido sin interrupciones y se origina un nuevo ítem cada vez que exista una interrupción debido a atascos, escritura en silencio, formulación de preguntas al investigador o a cualquier otra hecho que genere una interrupción en el transcurso de la resolución. Esta división inicial, supone un análisis ya que es necesario decidir, cuáles interrupciones serán tomadas en cuenta para fragmentar el discurso oral, además la mera colocación de los signos de puntuación en la trascripción escrita, de forma que se adecue, al menos en líneas generales, a las reglas de la gramática, es un análisis del texto oral producido por los sujetos (Puig, 1996).

Una adaptación del análisis de protocolos realizado por Schoenfeld (1985) fue utilizada como base para nuestro estudio. Schoenfeld, divide los protocolos de resolución de problemas en trozos macroscópicos de conducta constante llamados episodios. Un episodio es un período de tiempo durante el cual un resolutor o un grupo de resolutores está ocupado en una acción específica. Esta definición de episodio tiene que estar acompañada para que tenga sentido, de una definición correspondiente a cuales son los tipos de conducta distintas que se van a usar para calificarlos y segmentar así el protocolo en trozos. En nuestro análisis, en lugar de examinar, como lo hace Schoenfeld, las conductas de los estudiantes en la resolución de pro- 
blemas, se observó el empleo de las representaciones y la traducción entre representaciones por parte de los estudiantes cuando resuelven problemas de optimización. Al hablar de empleo de las representaciones, debemos tener presentes qué representaciones vamos a usar, así, para nuestro análisis tomaremos en cuenta tres tipos de representación: representación verbal del enunciado del problema, representaciones pictóricas y representaciones simbólicas, así como las traducciones entre ellas (ver fig. 2): de lo verbal a lo pictórico o viceversa, de lo pictórico a lo simbólico o viceversa, y de lo simbólico a lo verbal o viceversa.

Cada una de las representaciones y las traducciones entre representaciones constituiría un episodio; es decir, que se tenían seis episodios. Estos episodios establecen un segundo criterio para el análisis de los protocolos; para realizar este análisis se procedió a recorrer la trascripción previamente realizada cortando con líneas verticales cada vez que consideraba que un párrafo pertenecía a un episodio en particular.

Al efectuar esta división había párrafos que no se correspondían con ninguno de los seis episodios anteriores, viéndose la necesidad de anexar un séptimo episodio, en el cual se incluirían los eventos que no eran catalogados como representación. Es importante señalar que los episodios no tienen carácter lineal, es decir, un resolutor usa un episodio en particular en cualquier momento y puede estar "saltando" entre episodios a medida que resuelve el problema

Para facilitar la división del protocolo escrito, a los episodios antes mencionados le fueron anexados indicadores, de tal manera que las categorías de los episodios son descritas teórica y empíricamente (Artzt y Armour-Thomas, 1992). El marco para el análisis de protocolos de resolución de problemas que hemos construido para analizar las representaciones y la traducción entre representaciones (Villegas y Castro, 2002), consta de los episodios mostrados en la tabla 3.

Con este marco para el análisis de protocolos de resolución de problemas uno de los investigadores analizó las transcripciones generando un protocolo escrito el cual fue comprobado por otro investigador usando el mismo marco de análisis de protocolo. Cuando existía duda en ubicar un ítem dentro de un episodio en particular o no había concordancia entre los investigadores, ambos observaban de nuevo el vídeo y acordaban a que episodio pertenecía el ítem, incrementando así el grado de fiabilidad en el análisis. 


\section{Tabla 3. Marco para el análisis de protocolos}

Episodio 1: Representación interna del enunciado del problema.

Descripción: El estudiante lee el problema.

Indicadores:

a. El estudiante lee el problema en voz alta.

b. El estudiante, lee el problema en silencio ó "murmurando".

c. El estudiante enuncia el problema cambiando algunas palabras por otras propias de su lenguaje personal.

Episodio 2: Representación Pictórica.

Descripción: El estudiante realiza, opera y modifica representaciones pictóricas.

Indicadores:

a. El estudiante dibuja con papel y lápiz una representación pictórica ó bien modifica las representaciones previamente realizadas.

b. El estudiante opera con las representaciones pictóricas.

c. El estudiante señala u observa una representación pictórica ó bien verbaliza términos asociados a las representaciones pictóricas.

d. El estudiante presenta a través de movimientos corporales, bien sea con las manos u otras partes del cuerpo, representaciones pictóricas.

\section{Episodio 3: Representaciones Simbólicas.}

Descripción: El estudiante realiza, opera y modifica representaciones simbólicas.

Indicadores:

a. El estudiante resuelve ó intenta resolver con lápiz y papel una expresión simbólica.

b. El estudiante verbaliza como puede resolver una ecuación ó verifica como fue resuelta.

c. El estudiante modifica, reescribe ó elimina una expresión simbólica.

d. El estudiante observa ó señala una expresión simbólica.

\section{Episodio 4: Traducción entre una representación verbal y una representación Pictórica.}

Descripción: El estudiante relaciona de alguna forma una representación pictórica con una representación verbal.

Indicadores:

a. El estudiante realiza con papel y lápiz una representación pictórica directamente del enunciado, sin modificarlo ó modificándolo a su lenguaje personal.

b. El estudiante transforma ó modifica una representación pictórica de acuerdo a una nueva interpretación del enunciado.

c. El estudiante establece relaciones entre el enunciado y una representación pictórica, a través de verbalizaciones ó gesticulaciones.

d. El estudiante representa elementos pictóricos a través de movimientos corporales, especialmente con las manos, mientras lee el enunciado del problema. 
Episodio 5: Traducción entre una representación pictórica y una representación simbólica.

Descripción: El estudiante relaciona de alguna manera una representación pictórica y una representación simbólica.

Indicadores:

a. El estudiante formula una expresión ó parte de ella con lápiz y papel desde una representación pictórica, ó realiza una representación pictórica desde una expresión simbólica.

b. El estudiante establece relaciones entre una expresión simbólica y una representación pictórica a través de verbalizaciones ó gesticulaciones.

c. El estudiante realiza cambios ó elimina una representación pictórica previamente construida debido a resultados obtenidos simbólicamente.

d. El estudiante modifica ó elimina expresiones simbólicas debido a resultados obtenidos en representaciones pictóricas ó a una nueva representación pictórica.

e. El estudiante asigna símbolos a una representación pictórica.

\footnotetext{
Episodio 6: Traducción entre una representación simbólica y una representación verbal.

Descripción: El estudiante relaciona, de alguna manera, una representación simbólica con una representación verbal.

Indicadores:

a. El estudiante formula una expresión simbólica ó parte de ella del enunciado, bien sea sin modificarlo ó modificándolo a su lenguaje personal.

b. El estudiante transforma ó modifica una expresión simbólica debido a una nueva interpretación del enunciado.

c. El estudiante reformula el enunciado de una manera diferente debido a algún resultado obtenido en una expresión simbólica.

d. El estudiante asigna una variable a alguna parte del enunciado.

e. El estudiante relaciona una expresión simbólica con el enunciado a través de verbalizaciones ó gesticulaciones.
}

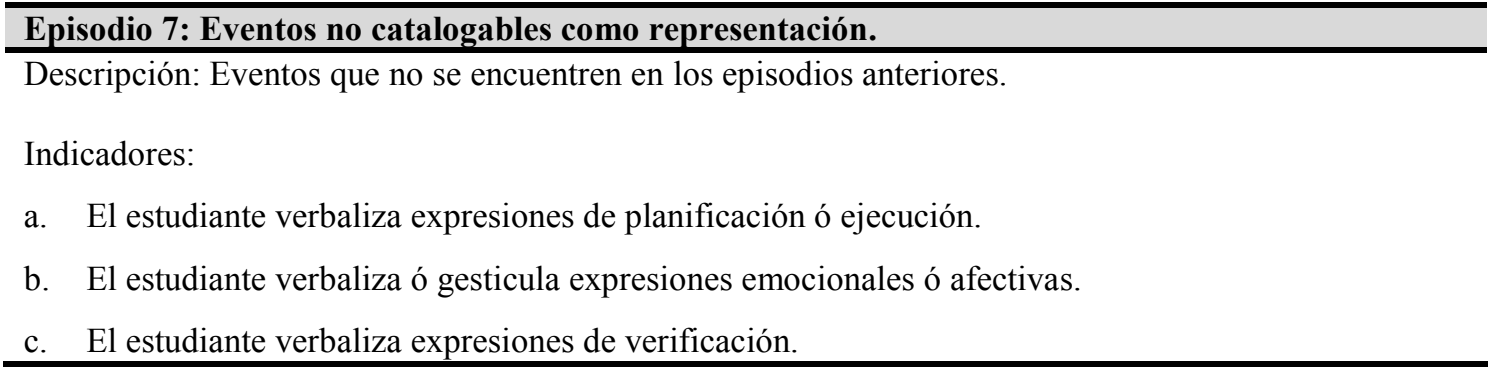

A este protocolo le fue anexado el trabajo escrito realizado por el resolutor en la sesión de resolución de problemas. Para hacer esto, el investigador observaba nuevamente el vídeo, e iba correlacionando, las verbalizaciones realizadas por el resolutor con el material escrito en 
la sesión de la resolución de problemas. Esto se intento hacer lo más parecido posible a lo que el resolutor había hecho en la sesión, de tal forma que al observar el documento, se "perciba" lo que el resolutor estaba realizando en cada instante de la resolución, es decir, que al ir leyendo las verbalizaciones producidas se sepa el momento exacto en que el resolutor escribía en el folio destinado a la resolución del problema. Aunado a esto, a la transcripción definitiva también se le colocó el tiempo en el que comenzaba y finalizaba cada ítem.

Por último, se anexaban también los gestos que acompañaban a los deícticos (este, aquí, así, etc.), los cuales son muy importantes para entender lo que los resolutores señalaban; para esto se dibujó una mano que indica con su dedo la parte de la resolución a la cual se está refiriendo. Para una mejor y más fácil lectura de los protocolos definitivos, utilizamos las siguientes convenciones:

1. Texto a la izquierda: verbalizaciones producidas por los resolutores.

2. Texto y gráficas a la derecha: trabajo escrito realizado por los resolutores en la sesión de resolución de problemas.

3. Texto entre corchetes: acciones realizadas por los resolutores e interpretadas por el investigador.

4. Texto en cursiva: intervenciones del investigador.

5. Puntos suspensivos (...): pausas en las verbalizaciones realizadas por el resolutor.

6. Tiempo (al principio y arriba de cada ítem): tiempo de inicio de cada episodio, por supuesto este tiempo, es el tiempo final del episodio anterior.

\begin{tabular}{|l|l|}
\hline $\begin{array}{l}\text { 01:29 } \\
\text {... Bueno vamos a ver si podemos cruzar el agua }\end{array}$ \\
\hline $\begin{array}{l}\text { 01:43 } \\
\text {.. entonces la mínima distancia por agua sería la hipotenusa, }\end{array}$ \\
\hline $\begin{array}{l}\text { 01:44 } \\
\text { la hipotenusa, }\end{array}$
\end{tabular}




\begin{tabular}{|l|c|}
\hline $\begin{array}{l}\text { 01:56 } \\
\text { la calculamos } 1^{2}+5^{2} \text { ser' an } 25 \text { y } 1 \text { 26 la hipotenusa, es } \\
\text { raiz de } 26\end{array}$ & $h^{2}=1^{2}+5^{2} \Rightarrow h=\sqrt{26}$ \\
\hline $\begin{array}{l}\text { 02:14 } \\
\text {.. bien, eh ..., si queremos echarlo por tierra ..., pues sería 1 de } \\
\text { agua esto sería una posibilidad, }\end{array}$ & \\
\hline $\begin{array}{l}\text { 02:32 } \\
\text { primera posibilidad solo por agua, }\end{array}$ & $1^{\circ} h^{2}=1^{2}+5^{2} \Rightarrow h=\sqrt{26}$ \\
\hline $\begin{array}{l}\text { 02:35 } \\
\text { segunda posibilidad eh, podemos hacerlo por agua y por tierra, } \\
\text { sería 1 Km por agua más 5 Km por tierra }\end{array}$ & $2^{\circ} 1 \mathrm{Km} .+5 \mathrm{Km}$. \\
\hline
\end{tabular}

\begin{tabular}{|l|c|}
\hline $\begin{array}{l}03: 24 \\
20 .\end{array} \quad$ entonces, el coste total será 3y euros, 5x euros & \multicolumn{1}{c|}{$\begin{array}{c}3 y € \\
\text { 03:52 }\end{array}$} \\
$\begin{array}{l}21 . \quad \text { en total son } 3 y+5 x, \text { ¿cuando es mínimo esta } \\
\text { función? }\end{array}$
\end{tabular}

$c=3 y+5 x=$ f minimo

Figura 3. Fragmento del protocolo del resolutor A resolviendo el problema 1.

\section{Resultados}

Los protocolos de cada participante fueron analizados, como se ha descrito en el apartado anterior y posteriormente los resultados fueron reportados en forma de análisis de caso, esto consiste en realizar una interpretación del trabajo efectuado por los resolutores. El análisis los presentamos en forma de narración con una serie de comentarios interpretativos y, soportado por algunos ítems ilustrativos. Para su elaboración se han tomado en cuenta, los protocolos escritos elaborados a partir del marco para el análisis de protocolos desarrollado en este estudio, los resultados obtenidos a partir del análisis de las tablas realizadas (Villegas, 2002) y la "revisión" de los trabajos escritos elaborados por los resolutores. En este análisis de caso se puede observar el comportamiento del resolutor frente al problema, su competencia en el uso de las representaciones, su habilidad para hacer traslaciones entre varias representaciones y cómo, la planificación, el monitoreo, el control y las "afectividades locales" (Goldin, 1998a) son generadores de éxito, o bien, creadores de obstáculos en la resolución de problemas. A continuación presentamos el análisis de caso correspondiente al resolutor $\mathrm{C}$ intentando resolver el problema 1. 
El resolutor comienza con un breve episodio de lectura; aunque siente incertidumbre si comenzar a resolver inmediatamente el problema -«¿puedo empezar a escribir ya, no?»- (2), este episodio fue definido como no catalogado como representación y en el se ven manifiestas verbalizaciones de tipo afectivo. Continúa con la lectura del problema, pero sin realizar ninguna traducción hacia otra representación. Cuando termina la lectura del enunciado del problema, ve la necesidad de realizar una traducción de la situación verbal a la pictórica -«voy a pintar» (4)- y comienza a realizar la traducción.

C realiza una representación pictórica directamente del enunciado, comienza a través del dibujo a representar la situación, verificando la relación que existe entre su "dibujo" y el enunciado -«anchura, corriente arriba» (7)-, simultáneamente a la verbalización señala con su mano el dibujo mientras lee el problema.

Exteriorizando un no entendimiento del problema planteado, $\mathrm{C}$ vuelve a leer gran parte del enunciado y al finalizar evidencia que conoce cómo resolver el problema y verbaliza una especie de plan - «esto es una mezcla entre tierra y agua, vale, una función de optimización» (9)-, aunque de lo que habla, por supuesto, no es una "función de optimización", se deduce que lo que quiere decir es que es un problema de optimización. Enseguida, comienza a asignar variables a las posibles formas de "tender el cable" y al final vuelve a leer la pregunta formulada en el enunciado -«... por metro ¿cuál será la trayectoria del tendido que resulta más económica?»(13)-, esto con el fin de asegurarse de no desviar su cometido.

C plantea la ecuación de la trayectoria del tendido directamente de la representación pictórica que había realizado -«la trayectoria del tendido será... $y+x$ metros, que sea mínimo» (14)- y aunque la plantea erróneamente ya que olvida considerar el precio del cable, demuestra destreza en la traducción entre la representación pictórica y la representación simbólica.

La ecuación formulada la relaciona inmediatamente con el enunciado usando palabras de su lenguaje personal -«esto es lo que me preguntaban, ¿cuanto es el $y+x$ que te haga la distancia mínima?» (15)-, como se puede observar existe una gran capacidad en el resolutor 
para realizar las traducciones entre representaciones, moviéndose apropiadamente entre las tres representaciones en un breve período de tiempo (menos de 20 segundos).

Paso siguiente, plantea la necesidad de relacionar la $x$ con la $y$ observando la figura realizada previamente -«vamos hallar una relación entre $x$ e $y$, donde $x$ es por tierra e $y$ es por agua» (16)- esto no es tan claro para él, entonces relaciona la figura con el enunciado buscando ideas que le sugieran el camino a seguir, no las encuentra, se desespera un poco - «.... ¡madre mía!, $x$ por metros, que sea mínimo» (18)-, se da cuenta de que los cables tienen diferentes precios y construye la función de coste (con los precios invertidos ¿culpa de los nervios?) y verbaliza una expresión de planificación -«.... tengo que $y$, si pongo $x$ en función de $y$, y optimizo, obtengo un mínimo, calculo cuanto vale $y$ en función de $x \gg(22)-$.

De la función de coste formulada: despeja $y$ en función de $x$-«obtengo que $y=-5 x / 3 »(23)-$, para hacer esto ¿supuso que el coste es cero?, ó ¿los nervios vuelven a jugar un papel fundamental?

\begin{tabular}{|l|c|}
\hline $\begin{array}{l}03: 24 \\
22 . \quad \text { entonces, el coste total será 3y euros, 5x euros }\end{array}$ & $\begin{array}{c}3 y € \\
\text { Coste total } 5 x €\end{array}$ \\
\hline $03: 52$ & $c=3 y+5 x=\mathrm{f}$ mínimo \\
$23 . \quad$ en total son $3 y+5 x$, ¿cuando es mínimo esta & \\
función?
\end{tabular}

Manifiesta de nuevo incertidumbre, recordando, primero y luego leyendo el enunciado del problema y además verbaliza relaciones entre una expresión simbólica y el enunciado buscando dos cosas: la solución del problema y lograr tranquilizarse.

C deriva la función de $y$ encontrada y la relaciona mentalmente con una representación pictórica, -«en donde el mínimo es una recta» (30)-, -«... el mínimo se alcanzaría ... rápidamente $\ldots y^{\prime}=-\frac{5}{3}, »-(31)$, cometiendo con lo anterior graves errores, de los cuales se percata rápidamente y se propone leer el problema de mejor manera -«leo despacio el problema» (33)-, lee de nuevo el problema, notándose esta vez una mayor concentración en la lectura.

Después de leer el problema, da un repaso a todo lo hecho anteriormente, tomando en cuenta la figura y las ecuaciones realizadas, y relacionándolas inmediatamente con el enun- 
ciado del problema para cerciorarse de que está haciéndolo bien. Después esto retoma la función coste, y la idea de relacionar la $x$ con la $y$,

\begin{tabular}{|c|c|}
\hline $\begin{array}{l}06: 36 \\
38 . \quad \text { si esto es } x \text {, esto es } 5-x, y \text { esto es } y\end{array}$ & 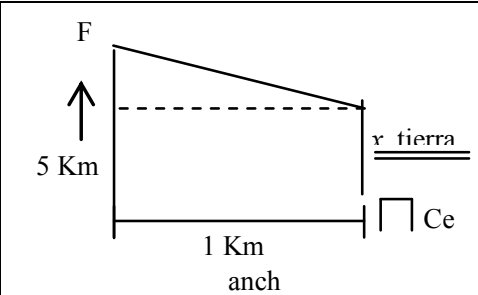 \\
\hline $\begin{array}{l}\text { 06:42 } \\
\text { 39. como esto es lo que quiero que sea mínimo }\end{array}$ & $c 3 y+5 x=\mathrm{f}$ mínimo \\
\hline $\begin{array}{l}\text { 06:44 } \\
\text { 40. voy a hallar la relación que existe entre } x \text { e y, }\end{array}$ & \\
\hline
\end{tabular}

El plan manifestado por C, lo conduce por buen camino y dice de él que, además de ser hábil en el manejo de las representaciones y las traducciones entre ellas tiene buen control y buena gerencia en la resolución de problemas.

Posteriormente, a través del uso de la figura realizada, establece la relación entre $x$ e $y$, $-«$ donde tengo que $y$ es igual a la raíz de $1+25,26+x^{2}-10 x »(44)-$, y cuando va a sustituir la relación encontrada en la función de coste, se da cuenta del error cometido al intercambiar los precios del cable y los corrige.

\begin{tabular}{|c|c|}
\hline $\begin{array}{l}\text { 08:06 } \\
\text { 48. } \quad \text { entonces el coste es igual a } 3 \text { que son los euros que vale por } \\
\text { metro, por tierra, }\end{array}$ & por tierra 3 euros $/$ metro \\
\hline $\begin{array}{l}08: 15 \\
49 . \quad \text { [murmura] llamamos } 3 \text { euros por metro esto es por } \\
\text { tierra no, esto sería } 3 x \text { y por agua cuesta } 5 \text {, esto sería } 5 y \text {, }\end{array}$ & \\
\hline $\begin{array}{l}08: 37 \\
50 . \quad \text { entonces tengo que la función de coste es } \\
3 x+5 \text { y esto es lo que quiero que sea mínimo, }\end{array}$ & $\begin{array}{rl}\mathrm{c}= & 3 x+5 y \underset{ }{\leftarrow} \\
\quad & \text { Coste total } \\
& \text { (Mínimo }) \\
5 x & €\end{array}$ \\
\hline
\end{tabular}


Sustituye la ecuación, opera simbólicamente con mucha tranquilidad hasta que cuando va a derivar parece que no es de su agrado el que una raíz sea parte de la función, - «la raíz se podría quitar, no?,» (54)-, disminuye la tranquilidad, se incrementa la inseguridad, - «bueno pero ya está, vamos a ver que sale» (55)-, deriva la función, simplifica mal, cuestión que no va afectar la consecución de la resolución del problema.

Continúa operando con representaciones simbólicas, siguiendo el patrón de "resuelvo, observo y/ó verifico, resuelvo, observo y/ó verifico" (llamemos a este patrón resoluciónverificación), tarea que es muy importante ya que realiza verificaciones inmediatas de los pasos que está efectuando.

A pesar de que C está haciendo buen uso de las representaciones y de la traducción entre representaciones, la no concentración y los nervios le han jugado una mala pasada en cálculos que, para él, deberían ser triviales. El camino hacia la solución se le ha complicado por los pequeños errores cometidos y $\mathrm{C}$ intenta buscar tácticas que lo encaminen, - «esta sería la raíz cuadrada, a ver, a ver, a ver, esto es demasiado desde mi punto de vista, intentaré darle la vuelta... porque tiene que dar $y$ en la ecuación, creo que voy a tener que despejar a $y »(68)$. Sigue con la pauta de revisión-verificación antes mencionada, la cual le lleva a una solución que, aunque difiere del resultado, es válida y aceptable desde varios puntos de vista: buen uso de las representaciones, habilidad en la traducción entre representaciones, planificación, verificación, entre otras.

\section{Discusión}

El análisis de caso anterior se realizó para cada uno de los resolutores intentando resolver cada uno de los problemas planteados, este tipo de análisis conjuntamente con el análisis de los tiempos y las frecuencias de uso de cada representación y la revisión de los trabajos escritos, los cuales se efectuaron en un trabajo más amplio (Villegas, 2002), nos sugirió que los tres sujetos participantes tienen una tipología diferente, de acuerdo a las representaciones y las traducciones entre representaciones usadas en la resolución de problemas de optimiza- 
ción; esto nos lleva a pensar que existen tipologías bien diferenciadas de resolutores. A continuación presentamos una descripción de los resolutores participantes en el estudio:

\section{Resolutor A:}

Estudiante medianamente competente en el uso de las representaciones y la traducción entre representaciones, ya que a pesar de que las usa bien, no se da cuenta fácilmente de las relaciones entre varias representaciones.

Invierte un tiempo considerable en darse cuenta de la información que aportan algunas traducciones y algunos datos y hechos intrínsecos al enunciado. No se da cuenta, de forma rápida, de la representación más apropiada a usar en un momento determinado y algunas veces se siente inseguro al usarla.

\section{Resolutor B:}

Estudiante incompetente en el uso de las representaciones y la traducción entre representaciones en la resolución de problemas de optimización. Con poca habilidad para realizar traducciones con precisión y eficiencia. Frecuentemente tiene dificultades en entender los enunciados de los problemas, extraer información de ellos y hacer traducciones desde el enunciado hacia cualquier otro sistema de representación.

A pesar de que ciertas veces tiene la información necesaria para resolver el problema carece de la habilidad necesaria para descubrir en "ciertas piezas" algunas claves o la información necesaria para resolver el problema global. Usa poco y, algunas veces, incorrectamente el álgebra simbólica.

\section{Resolutor C:}

Estudiante muy competente en la resolución de problemas de optimización. Con mucha habilidad para hacer traducciones entre varias representaciones con precisión y eficacia. Claro entendimiento del tipo de información que revela una traducción. Habilidad para darse cuenta de la relevancia de los datos, relaciones y hechos expresados en el enunciado del problema o revelados por una traducción o representación. Habilidad para extraer información de una representación y usarla en otro tipo de representación. Destreza en el trabajo con más de 
una representación y con capacidad de trabajo con más de dos representaciones el mismo tiempo.

Este estudiante verifica lo que señalan Lesh, Post y Behr (1987), "los buenos resolutores de problemas tienden a ser suficientemente flexibles en el uso de varios sistemas de representaciones, y que ellos instintivamente cambian a la representación más conveniente en cualquier momento del proceso de resolución (p.38).

\section{Conclusiones}

El propósito de este estudio era describir cómo los resolutores expertos emplean las representaciones y la traducción entre representaciones en la resolución de problemas de optimización, para tal fin, en principio, se desarrolló un marco teórico para análisis de protocolos, el cual es una adaptación del desarrollado por Schoenfeld (1985) y toma como episodios ${ }^{2}$ las representaciones y la traducción entre representaciones. En el proceso de segmentación y codificación nos percatamos que existían algunos segmentos que no correspondían con los episodios considerados al inicio, por tanto fue necesario agregarlos, estos episodios fueron considerados como "eventos no catalógables como representación" y comprendían verbalizaciones de planificación ó ejecución, de afectividad y de verificación.

Una vez desarrollado el marco, se le aplicó a los protocolos verbales, generados por los resolutores; los análisis de datos generados sugieren la viabilidad y utilidad de este marco para la investigación de la influencia de las representaciones en la resolución de problemas matemáticos ${ }^{3}$. Aun así, estamos conscientes de que el marco para el análisis de protocolos elaborado, necesita algunas modificaciones que incrementarían su utilidad.

Tomando en cuenta los análisis efectuados se encontró que los tres sujetos participantes tienen una tipología diferente, de acuerdo a las representaciones y las traducciones entre

\footnotetext{
${ }^{2}$ Períodos de tiempo durante los cuales un resolutor está ocupado en una acción específica.

${ }^{3} \mathrm{~A}$ pesar que el trabajo se hizo con problemas de optimización, creemos que, bajo ciertos, el marco es válido para otro tipo de problemas.

${ }^{4}$ Las imágenes de vídeo y el tono de voz de los resolutores, aportaron gran cantidad de información y creemos, que son necesarias en este tipo de investigaciones.
} 
representaciones usadas en la resolución de problemas de optimización, esto nos lleva a pensar que existen tipologías bien definidas de resolutores.

La caracterización de los resolutores, pone de manifiesto la fuerte relación que existe entre el éxito en la resolución de problemas de optimización y la habilidad en la construcción, empleo y articulación de las representaciones.

Consideramos que las actividades realizadas para la obtención de datos fueron amplias y profundas. La revisión de los trabajos escritos, los protocolos verbales, las imágenes de vi$\mathrm{deo}^{4}$, los protocolos escritos, las tablas de tiempo y frecuencia, permiten obtener un conocimiento profundo de cada resolutor. Asimismo, la presentación de los protocolos escritos, además de darnos información, es "fácil" y cómoda de leer e interpretar, de tal forma que otros investigadores pueden extraer de ellos sus propias impresiones, además, la metodología usada permite conocer en cada momento cuales son las representaciones y las traducciones entre representaciones manifiestas por el resolutor, cuando intenta resolver problemas de optimización y si es hábil en el empleo de las mismas.

Somos consientes de que el estudio realizado no da sustento para establecer tipologías de sujetos, pero proporciona una aproximación inicial para las investigaciones que se quieran realizar en esta dirección. Consideramos necesario que se continúen realizando investigaciones donde podamos observar la influencia de las representaciones en la resolución de problemas, sobre todo en aquellos problemas en la cual la representación de partida es un enunciado en la lengua natural. 


\section{Referencias}

Anguera, M. T., Arnau, J., Ato, M., Martínez, R., Pascual, J., \& Vallejo, G. (1995). Métodos de investigación en Psicología. Madrid: Síntesis.

Artzt, A. F., y Armour-Thomas, E. (1992). Development of a cognitive-metacognitive framework for protocol analysis of mathematical problem solving in small groups. Cognition and Instruction, 9(2), 137-175.

Ballard, J. (2000). Student's use of multiple representations in mathematical problem solving. (Doctoral Dissertation, Montana State University-Bozeman, 2000). UMI: 9989359.

Brenner, M. E., Mayer, R. E., Moseley, B., Brar, T., Durán, R., Reed, B. S., \& Webb, D. (1997). Learning by understanding: The role of multiple representations in learning algebra. American Educational Research Journal, 37(4), 663-689.

Campos, M. A., y Estrada, J. (1999). Representaciones matemáticas de estudiantes preuniversitarios en la resolución de un problema de optimización. Educación Matemática, $\mathrm{XI}(2), 30-50$.

Castro, E. (2008). Resolución de problemas. Ideas, tendencias e influencias en España. En R. Luengo, B. Gómez, M. Camacho y L.J. Blanco (Eds.), Investigación en Educación Matemática XII (pp. 113-140). Badajoz: SEIEM

Castro, E., Morcillo, N. y Castro, E. (1999). Representations Produced by Secondary Education Pupils in Mathematical Problem Solving. En F. Hitt, \& M. Santos (Eds.), Proceedings of the Twenty First Annual Meeting of the North American Chapter of the International Group for the Psychology of Mathematics Education. Vol 2. (pp. 547558). Columbus, OH: ERIC Clearinghouse for Science, Mathematics, and Environmental Education.

Cifarelli, V. (1998). The development of mental representations as a problem solving activity. Journal of Mathematical Behaviour, 17(2), 239-264.

Cuoco, A.A, y Curcio, F. R. (Eds.) (2001). The roles of representation in school mathematics. Reston, Virginia: The National Council of Teachers of Mathematics.

Dane, F. C. (1990). Research methods. Pacific Grove, CA: Brooks/Cole Publishing.

DeBellis, V.A., y Goldin, G.A. (2006). Affect and meta-affect in mathematical problem solving: a representational perspective. Educational Studies in Mathematics, 63, 131-147.

Dreyfus, T (1991). Advanced mathematical thinking processes. En D. Tall (Ed.). Advanced mathematical thinking (pp. 25-41). Netherlands: Kluwer Academic Publishers.

Duval, R. (1993). Semiosis y noesis. En lecturas en didáctica de las matemáticas: Escuela francesa (pp. 118-144). México: Sección de matemática educativa del Cinvestav-IPN. 
Duval, R. (1998). Registros de representación semiótica y funcionamiento cognitivo del pensamiento. En F. Hitt (E) Investigaciones en matemática educativa II, (pp. 173-201). México: Grupo Editorial Iberoamérica.

Duval, R. (2006). A cognitive analysis of problems of comprehension in a learning of mathematics. Educational Studies in Mathematics, 61, 103-131.

Ericsson, K.A. y Simon, H.A. (1993). Protocol analysis. Verbal reports as data. Cambridge, MA: MIT Press.

Ginsburg, H. P., Kossan, N. E., Schwartz, R., y Swanson, D. (1983). Protocol methods in research on mathematical thinking. En H. Ginsburg (Ed), The development of mathematical thinking (pp. 7-47). New York: Academic press..

Goldin, G. (1998a). Representational systems, learning, and problem solving in mathematics. Journal of Mathematical Behaviour, 17(2), 137-165.

Goldin, G. (1998b). The PME Working Group on representations. Journal of Mathematical Behaviour, 17(2), 283-301

Gutiérrez, J., Pozo, T., y Fernández, A. (2002). El estudio de caso en la lógica de la investigación interpretativa, Revista Arbor: Ciencia, Pensamiento y Cultura, $\mathrm{n}^{\mathrm{0}}$ 675, tomo CLXXI, pp. 533-558.

Hiebert, J., y Camperter, T. (1992). Learning and teaching with understanding. En D.A. Grows (Ed.), Handbook of research on mathematics teaching and learning (pp.65-97). New York: MacMillan Publishing Company.

Hitt, F (1996). Sistemas semióticos de representación del concepto de función y su relación con problemas epistemológicos y didácticos. En F. Hitt (Ed.), Didáctica: Investigaciones en Matemática Educativa (pp. 245-264). México: Grupo Editorial Iberoamericana.

Hitt, F. (Ed.) (2002). Representations and mathematical visualization. PME-NA Working Group (1998-2002). Mexico City: Cinvestav-IPN.

Janvier, C. (1987). Translation processes in mathematics education. En C. Janvier (Ed.), Problems of representation in the teaching and learning of mathematics (pp. 27-32). Hillsdale, NJ: Lawrence Erlbaum Associates.

Junta de Andalucia (1989). Diseños curriculares de matemáticas: Etapa 16-18. Sevilla: Conserjería de Educación y Ciencia.

Kaput, J. J. (1987). Representation systems and mathematics. En C. Janvier (Ed.), Problems of representation in the teaching and learning of mathematics (pp. 19-26). Hillsdale, NJ: Lawrence Erlbaum. 
Koedinger, K. R., y Nathan, M. J. (2004). The Real Story Behind Story Problems: Effects of Representations on Quantitative Reasoning. The Journal of the Learning Sciences, 13(2), 129-164.

Lesh, R., Post, T., y Behr, M., (1987). Representations and translations among representations in mathematics learning and problem solving. En C. Janvier (Ed.), Problems of representation in the teaching and learning of mathematics (pp. 33-40). Hillsdale, NJ: Lawrence Erlbaum Associates.

Marcelo, C. (Coord) (1991). El estudio de caso en la formación del profesorado y la investigación didáctica. Sevilla: Universidad de Sevilla.

NCTM (1989). Curriculum and evaluation standards for school mathematics. Reston, VA: El autor.

NCTM (2000). Principles and standards for school mathematics. Reston, VA: El autor.

NCTM (2006). Curriculum Focal Points for Prekindergarten through Grade 8 Mathematics. Reston, VA: El autor.

Porzio, D. (1999), Effects of differing emphases in the use of multiple representations and technology on students' understanding of calculus concepts. Focus on the Learning Problems in Mathematics, 21(3), 1-29.

Puig, L (1996). Elementos de resolución de problemas. Granada: Comares.

Rico, L. (2000). Sobre las nociones de representación y comprensión en la investigación en educación matemática. En L. C. Contreras, J. Carrillo, N. Climent, \& M. Sierra (Eds.), Cuarto Simposio de la Sociedad Española de Investigación en Educación Matemática. Huelva: Servicio de Publicaciones de la Universidad de Huelva.

Schoenfeld, A. H. (1985). Mathematical problem solving. New York, NY: Academic Press.

Schultz J. E., y Waters M. S. (2000). Why representation? Mathematics Teacher, 3(6), 448453.

Stake, R. E. (1999). Investigación con estudio de casos. Madrid: Morata.

Villegas, J. L. (2002). Representaciones en resolución de problemas: Un marco de análisis de protocolos. Trabajo de Investigación Tutelada. Granada: Dpto. Didáctica de la Matemática. Universidad de Granada.

Villegas, J. L., y Castro, E (2002). Marco para el análisis de protocolos de resolución de problemas de optimización. En J. M Cardeñoso, E. Castro, A. Moreno, \& $\mathrm{M}^{\mathrm{a}}$ Peñas (Eds.), Resolución de Problemas. Granada: Dpto. de Didáctica de la Matemática. Universidad de Granada. 